\title{
Improvement of Method to Estimate Colors of Amber
}

\author{
Hina Ishikawa ${ }^{a}$, Yoichi Kageyama, ${ }^{a}{ }^{,}$, Chikako Ishizawa $^{\mathrm{a}}$, Tsuyoshi Takahashi ${ }^{\mathrm{a}}$, Makoto Nishida ${ }^{\mathrm{b}}$, Hisao Shinden ${ }^{\mathrm{c}}$, \\ Toshio Takizawa ${ }^{\mathrm{c}}$, Taro Koyama ${ }^{\mathrm{c}}$, Makoto Kobushi ${ }^{\mathrm{c}}$ \\ ${ }^{a}$ Akita University, 1-1 Tegata Gakuen-machi, Akita-shi, Akita 010-8502, Japan \\ ${ }^{\mathrm{b}}$ The Open University of Japan, 1-1 Tegata Gakuen-machi, Akita-shi, Akita 010-8502, Japan \\ ${ }^{c}$ Kuji Kohaku Co., Ltd., 19-156-133 Kokuji-cho, Kuji-shi, Iwate 028-0071, Japan \\ *Corresponding Author: kageyama@ie.akita-u.ac.jp
}

\begin{abstract}
In a previous study, we demonstrated the color features of ambers and a method to estimate amber colors. We specifically focused on the red, green, blue (RGB) color space and the hue, saturation, value (HSV) color space. We proposed a method to estimate amber in four colors (yellow, brown, black, and ambers with an outer cover (AOCs) created with solid resin or amber dirt). However, due to the presence of ambers with partially exposed outer covers (APEOCs), the estimation accuracy of AOCs was $57.1 \%$. In this paper, we therefore demonstrate the color features of ambers and a method aimed at improving the estimation accuracy of AOCs. Specifically, we focus on five kinds of ambers: three colors (yellow, brown, and black), AOCs and APEOCs are examined. The proposed method consists of masking, detection of AOC, estimation of amber color, and detection of APEOC. Experimental results for 185 ambers show that the proposed method can estimate amber color with an accuracy of $96.7 \%$. It is also shown that an estimation accuracy of $91.4 \%$ for AOC color estimations is achieved.
\end{abstract}

Keywords: amber, color, RGB, HSV

\section{Introduction}

Amber is derived from resin, the composition of which can vary vastly; insects, leaves, flowers, and bark can be embedded in the amber for up to hundreds of millions of years, creating a very rare appearance that is also of academic and scientific importance [1]. The city of Kuji in Japan is one of the world's great producers of amber, and raw amber is processed into its finished form here. It is important to determine the color and quality of amber depending on its application; however, this discrimination is currently done with human eyes. A new compound, named kujigamberol, has been isolated from amber [2]. Further, regenerated amber, with $100 \%$ purity, has been made from pieces of amber [1]. Therefore, it is necessary to develop a method for estimating the type of color in amber debris, which in turn will contribute to cost reduction and high-speed processing. In a previous study, we demonstrated the color features of ambers and a method to estimate amber in four colors (yellow, brown, black, and ambers with an outer cover (AOCs) created with solid resin or amber dirt) [3]. However, due to the presence of ambers with partially exposed outer covers (APEOCs), the estimation accuracy of AOCs was $57.1 \%$. In this paper, we therefore demonstrate the color features of ambers and a method aimed at improving the estimation accuracy of AOCs.

\section{Data Acquisition Environment and Color Features}

The data was acquired in a dark room with two LED lights. It includes photographs taken with light falling from above (top-light images) and photographs taken with light coming from the bottom (bottom-light images). The top-light images have been taken with five different illumination levels (luminance levels 500-2500 lx, in stages of $500 \mathrm{~lx}$ ). The bottom-light images have been taken with nine different illumination levels (luminance levels 500-2500 lx, in stages of $250 \mathrm{~lx}$ ). Fig. 1 shows the environment where the photographs were taken. The study focuses on five kinds of amber: three colors (yellow, brown, and black), ambers with an outer cover (AOCs) created with solid resin or amber dirt, and ambers with partially exposed outer covers (APEOCs). Fig. 2 shows the training data used in this study.

For the experiment, 185 ambers (yellow amber: 70, brown amber: 40, black amber: 40, and AOC: 35 ) were used. In addition, we use 260 images (RGB color images; 


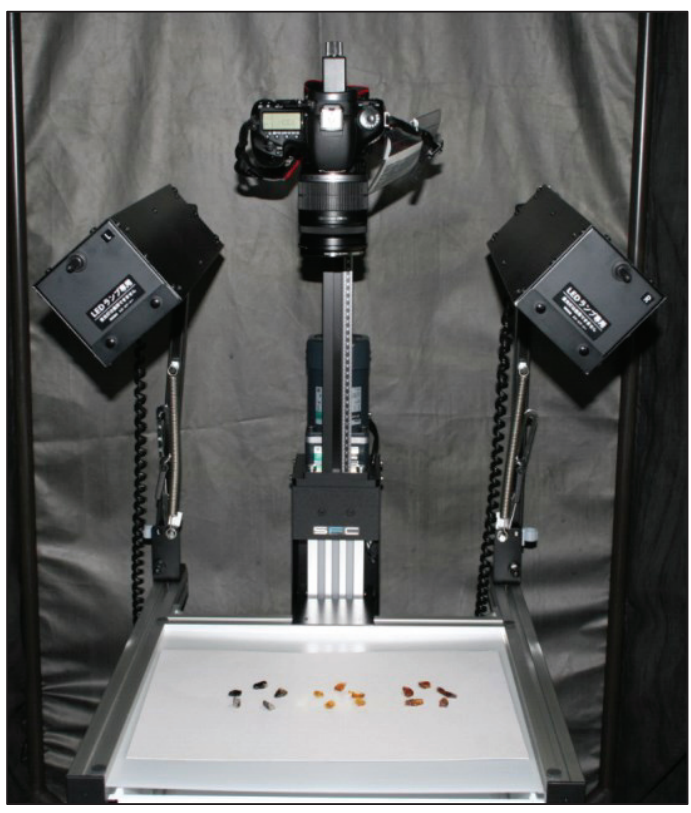

Fig. 1 Photography setup

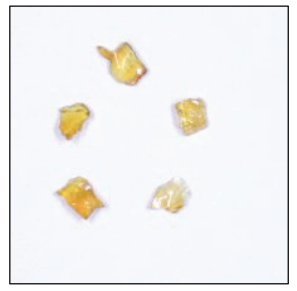

(a) Yellow

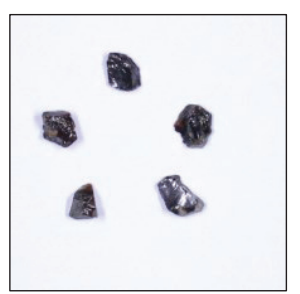

(c) Black

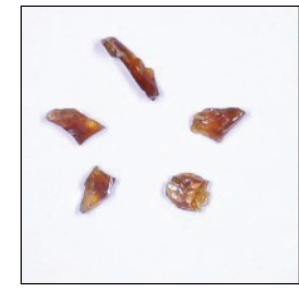

(b) Brown

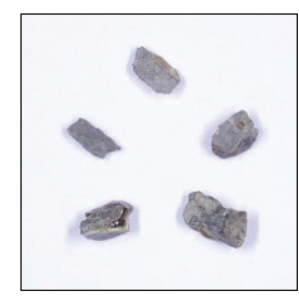

(d) $\mathrm{AOC}$

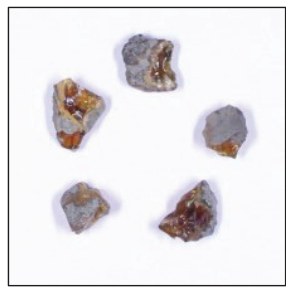

(e) APEOC

Fig. 2 Five kinds of amber sets used in the training data

$5184 \times 3456$ pixels) of amber (185 top-light images and 75 bottom-light images). The top-light images consisted of 70 images of yellow amber, 40 images of brown amber, 40 images of black amber, and 35 images of AOCs. The bottom-light images consisted of 40 images of brown amber and 35 images of AOCs.
In a previous study, we focused on the hue $(\mathrm{H})$ component of the hue, saturation, and value (HSV) color space and the red (R) component of the red, green, blue (RGB) color space as features useful for estimating the colors of amber [3].

In previous experiments, the mode value of the $\mathrm{H}$ component has shown there was little overlap between the three colors of ambers except AOCs. The findings indicated that the average value of the $\mathrm{R}$ component can be used to distinguish between yellow amber and the other ambers. The average value of the S component of AOCs was lower than that of the other ambers. It was also found that the average value of the $\mathrm{V}$ component was quite similar to the average value of the $\mathrm{R}$ component. The findings indicate that the value of the $\mathrm{R}$ component in the amber area was the largest because the $\mathrm{V}$ component was defined as the largest component of the RGB color space.

In the conventional method, color estimation was done using the above results.

Furthermore, in this study, we focused the variance value of the $\mathrm{H}$ component as a feature function for estimating APEOCs. Fig. 3 shows that the variance value of the $\mathrm{H}$ component of APEOCs and brown amber is lower than the variance value of the other ambers. This finding suggests that the variance value of the $\mathrm{H}$ component can be used to distinguish APEOCs and brown amber from other ambers.

\section{Proposed Method}

In our previous study, we proposed a method (the conventional method) for estimating amber to classify them into four kinds (yellow, brown, black, and AOC) [3]. The conventional method consists of masking, detection of AOC, and estimation of the amber color. Fig. 4 shows a flowchart of the conventional method. In the proposed method, APEOC detection has been added to the conventional method to improve the estimation accuracy of AOCs. This process uses top-light and bottom-light images. Fig. 5 shows a flowchart of the proposed method.

\subsection{Masking}

For top-light images, masking is done and the amber area is extracted. The masking comprises gray-scaling, binarization, expansion, and contraction processing with 10 operations, followed by color inversion [4]. 


\subsection{Detecting AOCs}

To detect AOCs, RGB values and HSV values are obtained from the amber area. The proportion of pixels with $\mathrm{R}=\mathrm{V}$ is calculated in the amber area. When the resulting value is 0.75 , with good results obtained between 0.70 and 0.90 for every step of 0.05 or more, it is judged as an amber.

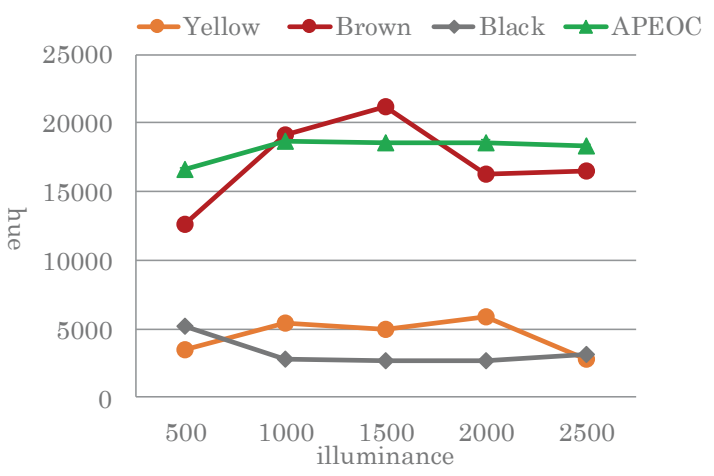

Fig. 3 Relation between hue and illuminance

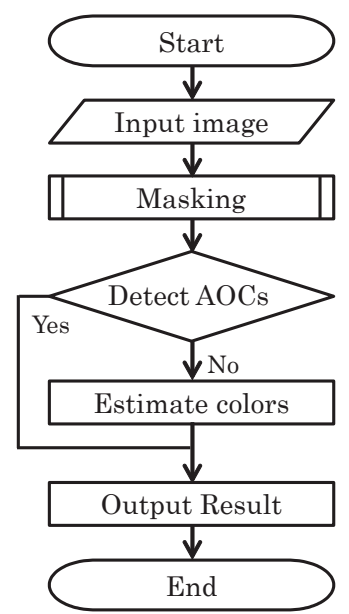

Fig. 4 Flowchart of the conventional method

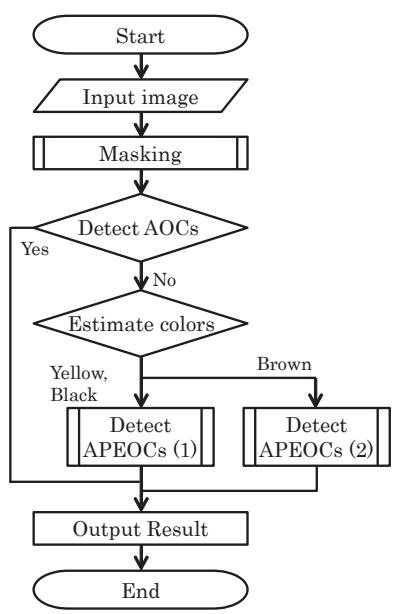

Fig. 5 Flowchart of the proposed method
Furthermore, the average value of the $\mathrm{S}$ component in the amber area of the target image is also calculated. When the calculated average value is above the threshold value, it is added to the list of ambers. Here, the threshold of the $\mathrm{S}$ component was set to 32.9 because good results were obtained for $73 \%$ of the average value of black ambers. The rest were classified as AOCs.

\subsection{Estimating Amber Colors}

In order to estimate the colors of the ambers, two degrees of attribution ( $\alpha$ and $\beta$ ) are calculated to estimate colors.

(a) Degree of Attribution $\alpha$

The degree of attribution $\alpha$ is calculated using equation (1). The average of the $\mathrm{R}$ component in the reference data is $\mathrm{A}$, and the average of the $\mathrm{R}$ component in the amber area is $\mathrm{B}$. The tolerance is C. Table 1 lists the preset values for calculating the degree of attribution $\alpha$.

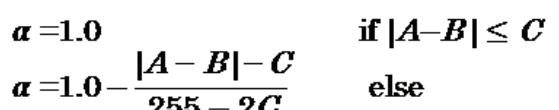

(b) Degree of Attribution $\beta$

The degree of attribution $\beta$ is calculated using equation (2). The mode value of the $H$ component in the reference data is $\mathrm{A}$, and the mode value of the $\mathrm{H}$ component in the amber area is B. The tolerance is C. Table 2 lists the preset values for calculating the degree of attribution $\beta$.

$$
\begin{array}{ll}
B=1.0 & \text { if }|A-B| \leq C \\
B=1.0-\frac{|A-B|-C}{(360-2 C) \div 2} & \text { else }
\end{array}
$$

(c) Membership of Each Amber Category

The degree of membership $\gamma$ for each amber category (yellow, brown, and black) is determined from the above two degrees and equation (3). The target image is also estimated as the amber color having the highest value.

$$
\gamma=0.5 \alpha+0.5 \beta
$$

\subsection{Detecting APEOCs}

(a) Detecting APEOCs from Yellow Amber and Black Amber

When the target image is estimated to be yellow amber or black amber, the variance value of the $\mathrm{H}$ component in the amber area is calculated. If the calculated variance value is above the threshold, it is added to the APEOC set. In this study, since good results have been obtained, the yellow amber threshold value was set to 
Table 1 The preset values for calculating the degree of attribution $\alpha$

\begin{tabular}{rrrr}
\hline & Yellow & Brown & Black \\
\hline $\mathrm{A}$ & 237 & 148 & 114 \\
\hline $\mathrm{C}$ & \multicolumn{3}{c}{15} \\
\hline
\end{tabular}

Table 2 The preset values for calculating the degree of attribution $\beta$

\begin{tabular}{rrrr}
\hline & Yellow & Brown & Black \\
\hline $\mathrm{A}$ & 43 & 358 & 249 \\
\hline $\mathrm{C}$ & \multicolumn{3}{c}{30} \\
\end{tabular}

15000 and the black amber threshold value was set to 9000 in advance. The rest were classified as yellow amber and black amber.

(b) Detecting APEOCs from Brown Amber

For target images estimated to be brown amber, masking is done in the conventional method for bottom-light images of the same amber, and the amber area is extracted. Furthermore, masking with mean shift [5] is done for the amber area, and the AOC area is extracted. A target image having many AOC regions is added to the APEOC set. In this study, the threshold of the area was set to $60 \%$.

\section{Experimental Results of Amber Estimation}

In order to verify the usefulness of the proposed method, we conducted an experiment to estimate 185 ambers. A parallel experiment was also conducted to estimate 185 ambers using the conventional method.

When carrying out these experiments, we first selected the optimum illuminance of the data set. As a result, the proposed method describes the results using top-light images at about $1500 \mathrm{~lx}$ and bottom-light images at about 750 lx. Table 3 shows the results of the amber estimation using the proposed method, and Table 4 shows the results of the amber estimation using the conventional method. Fig. 6 shows the results of the estimation. The proposed method successfully distinguishes 179 out of 185 ambers (96.7\%). This result shows an improvement of accuracy of $6.4 \%$ over the conventional method. Furthermore, by applying the APEOC detection technique of the proposed method, an APEOC estimated to be amber by the conventional method is estimated to be an AOC (Fig. 7 and Fig. 8). As a result, the estimation accuracy of AOCs has been improved by $31.4 \%$ compared with the conventional method.
The average processing time per image was calculated. The experimental environment consisted of a PC with Windows 7, Intel (R) Core (TM) i7-6700K @ 3.40 GHz CPU, and $8 \mathrm{~GB}$ of RAM. The processing time was about $0.173 \mathrm{~s}$.

Table 3 Estimation results of the proposed method

\begin{tabular}{|c|c|c|r|}
\hline Amber & Number of pieces & Success & Accuracy(\%) \\
\hline Yellow & 70 & 70 & $100.0 \%$ \\
\hline Brown & 40 & 39 & $97.5 \%$ \\
\hline Black & 40 & 38 & $95.0 \%$ \\
\hline AOC & 35 & 32 & $91.4 \%$ \\
\hline Total & 185 & 179 & $96.7 \%$ \\
\hline
\end{tabular}

Table 4 Estimation results of the conventional method

\begin{tabular}{|c|c|c|r|}
\hline Amber & Number of pieces & Success & Accuracy(\%) \\
\hline Yellow & 70 & 70 & $100.0 \%$ \\
\hline Brown & 40 & 39 & $97.5 \%$ \\
\hline Black & 40 & 38 & $95.0 \%$ \\
\hline AOC & 35 & 20 & $57.1 \%$ \\
\hline Total & 185 & 167 & $90.3 \%$ \\
\hline
\end{tabular}

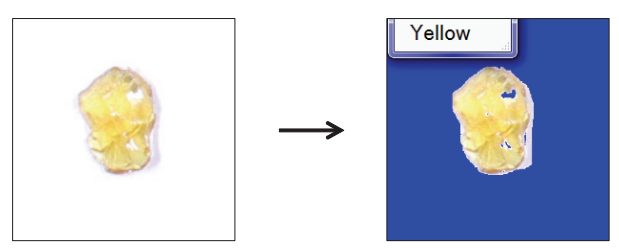

(a) Yellow amber

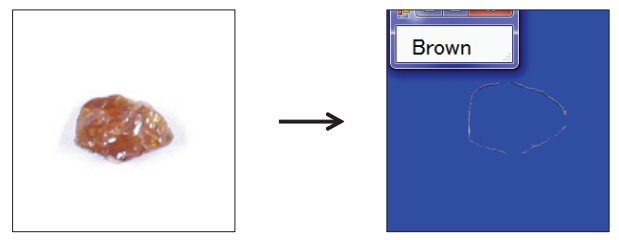

(b) Brown amber

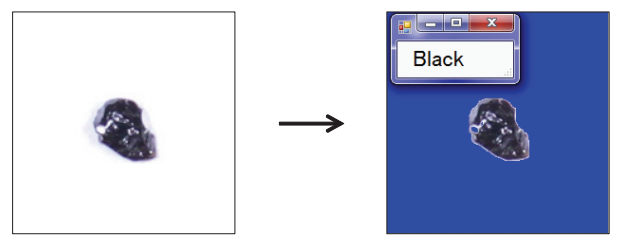

(c) Black amber

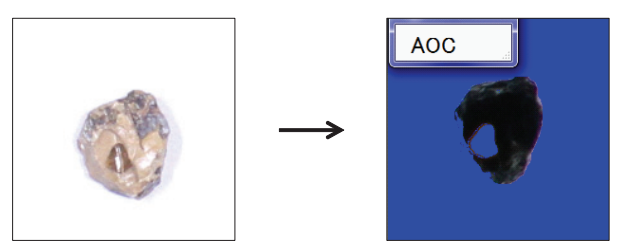

(d) $\mathrm{AOC}$

Fig. 6 Estimation results of amber using the proposed method 


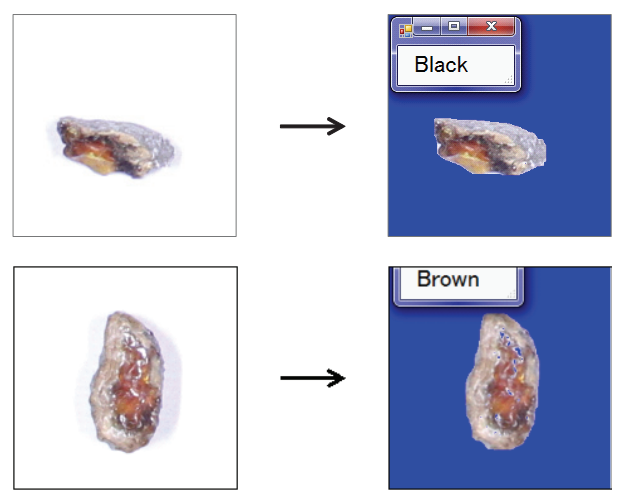

Fig. 7 Estimation results of APEOCs using the conventional method

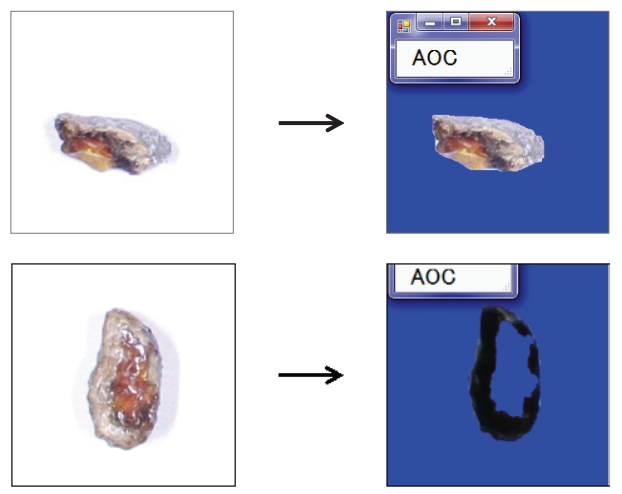

Fig. 8 Estimation results of APEOCs using the proposed method

\section{Conclusions}

In this study, we proposed a method to estimate amber for the purpose of improving the estimation accuracy of AOCs. In order to verify the usefulness of the proposed method, we conducted an experiment to estimate 185 ambers using the proposed method and the conventional method. Experimental results showed that the proposed method is useful for estimating amber. In particular, it was shown that the estimation accuracy of AOCs using the proposed method has improved with the help of APEOC detection compared with the conventional method.

The above conclusions suggest that the proposed method is useful as a method to estimate the colors of amber.

\section{Acknowledgment}

This research was supported by the Matching Planner Program (MP28116808546) of the Japan Science and Technology Agency (JST).

\section{References}

(1) Kuji Kohaku official site: http://www.kuji.co.jp/ Accessed June 12, 2018

(2) K. Kimura, et al.: "Kujigamberol, a new dinorlabdane diterpenoid isolated from 85 million years old Kuji amber using a biotechnological assay," Fitoterapia, Vol.83, No.5, pp.907-912 (2012)

(3) Y. Kageyama, H. Ishikawa, C. Ishizawa, T. Takahashi, M. Nishida, H. Shinden, T. Takizawa, T. Koyama, M. Kobushi: Method to Estimate Colors of Amber Debris," IEEJ Transactions on Electrical and Electronic Engineering, Vol.13, No.8, pp.1212-1213 (2018)

(4) M. Takagi, and H. Shimoda: Handbook of Image Analysis [Revised Edition], University of Tokyo Press (2004)

(5) Image Information Education Promotion Association: Digital Image Analysis [Revised Edition], Image Information Education Promotion Association (2015) 